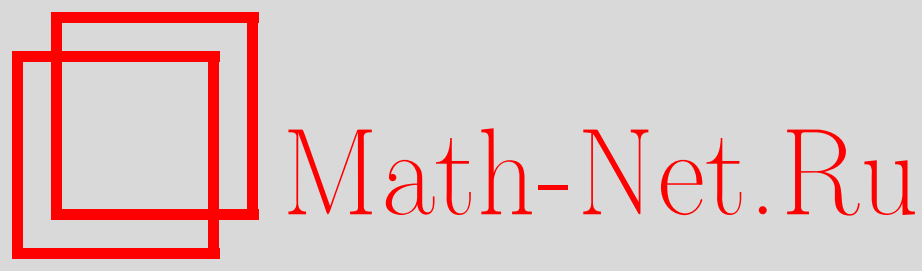

А. М. Зубков, К. А. Колесникова, Цепь Маркова с теоретико-числовым предельным распределением, Дискрет. матем., 2015, том 27, выпуск 3, 17-24

DOI: https://doi.org/10.4213/dm1332

Использование Общероссийского математического портала Math-Net.Ru подразумевает, что вы прочитали и согласны с пользовательским соглашением http://www . mathnet.ru/rus/agreement

Параметры загрузки:

IP: 3.81 .55 .215

26 апреля 2023 г., 13:25:19

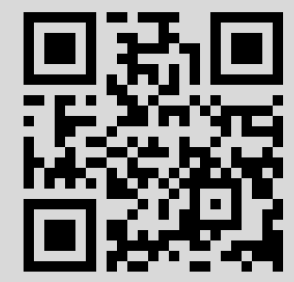




\title{
Цепь Маркова с теоретико-числовым предельным распределением
}

\author{
() 2015 г. А. М. Зубков*, К. А. Колесникова**
}

Пусть в урне находятся шары белого и черного цветов. За 1 шаг с вероятностями, равными $\frac{1}{2}$, либо число белых шаров увеличивается на число черных шаров, либо число черных шаров увеличивается на число белых шаров. Получены формулы для первых двух моментов общего числа шаров в урне, доказано, что предел функции распределения доли числа белых шаров в урне совпадает с теоретико-числовой функцией Минковского.

Исследование выполнено за счет гранта Российского научного фонда (проект 14-50-00005).

Ключевые слова: дискретные цепи Маркова, итерации случайных отображений, предельное распределение, функция Минковского.

\section{Введение}

Пусть $\left\{\xi_{t}=\left(\xi_{t, 1}, \xi_{t, 2}\right), t=0,1, \ldots\right\}$ - однородная по времени цепь Маркова с множеством состояний $\mathbb{N}^{2}=\{1,2, \ldots\}^{2}$ и переходными вероятностями

$$
\mathbf{P}\left\{\xi_{t+1}=\left(b_{1}, b_{2}\right) \mid \xi_{t}=\left(a_{1}, a_{2}\right)\right\}= \begin{cases}\frac{1}{2}, & \text { если }\left(b_{1}, b_{2}\right)=\left(a_{1}, a_{1}+a_{2}\right), \\ \frac{1}{2}, & \text { если }\left(b_{1}, b_{2}\right)=\left(a_{1}+a_{2}, a_{2}\right), \\ 0 & \text { в остальных случаях. }\end{cases}
$$

Цепь $\left\{\xi_{t}\right\}$, очевидно, невозвратна; ее траектории при $t \rightarrow \infty$ с вероятностью 1 уходят в бесконечность, монотонно не убывая по каждой компоненте. Их можно рассматривать как реализацию итерационного применения к начальному состоянию $\xi_{0}$ случайного отображения $\mathbb{N}^{2} \rightarrow \mathbb{N}^{2}$, определенного формулой (1). Эта последовательность связана с изучавшимся в [3], [5], [4] алгоритмом мультипликативных замен для построения цепи Маркова на множестве наборов порождающих элементов конечной группы $G$; в частном случае, когда $G$ коммутативна и порождается двумя элементами, пара $\left(\xi_{t, 1}, \xi_{t, 2}\right)$ определяет степени в произведении, выражающем изменяемый на $t$-м шаге элемент порождающего набора через элементы исходного

* Место работы: Математический институт им. В. А. Стеклова РАН, e-mail: zubkov@mi.ras.ru

** Место работы: Математический институт им. В. А. Стеклова РАН, e-mail: koleska@mi.ras.ru 
набора. В этой статье мы ограничились случаем $n=2$, поскольку в нем удается получить более явные результаты, чем в случае произвольного $n>2$.

В п. 1 выводятся формулы для первых двух моментов суммы компонент вектора $\xi_{t}$, в п. 2 рассматривается отношение $\gamma_{t}=\frac{\xi_{t, 1}}{\xi_{t, 1}+\xi_{t, 2}}$. Последовательность $\left\{\gamma_{t}\right\}$ образует цепь Маркова с множеством состояний $[0,1]$, доказано, что функция распределения $\gamma_{t}$ при $t \rightarrow \infty$ сходится к теоретико-числовой функции Минковского $?(x)$, введенной им в 1904 г. (см. [1]).

\section{1. Моменты суммы компонент}

Положим $\left|\xi_{t}\right|=\xi_{t, 1}+\xi_{t, 2}$.

Лемма 1. Справедливъ формуль

$$
\begin{gathered}
\mathbf{M}\left|\xi_{t}\right|=\left(\frac{3}{2}\right)^{t} \mathbf{M}\left|\xi_{0}\right| \\
\mathbf{M}\left|\xi_{t}\right|^{2}=\frac{\left(\lambda_{1} \lambda_{2}^{t}-\lambda_{2} \lambda_{1}^{t}\right) \mathbf{M}\left|\xi_{0}\right|^{2}+\left(\lambda_{1}^{t}-\lambda_{2}^{t}\right) \mathbf{M}\left|\xi_{1}\right|^{2}}{\lambda_{1}-\lambda_{2}}
\end{gathered}
$$

где $\lambda_{1}=\frac{5+\sqrt{17}}{4} \approx 2,2808, \lambda_{2}=\frac{5-\sqrt{17}}{4} \approx 0,2192$.

Доказательство. Так как при любом $t \geqslant 1$

$$
\mathbf{P}\left\{\left|\xi_{t}\right|=\left|\xi_{t-1}\right|+\xi_{t-1,1}\right\}=\mathbf{P}\left\{\left|\xi_{t}\right|=\left|\xi_{t-1}\right|+\xi_{t-1,2}\right\}=\frac{1}{2},
$$

то по формуле полного математического ожидания

$$
\mathbf{M}\left|\xi_{t}\right|=\frac{1}{2}\left(\mathbf{M}\left(\left|\xi_{t-1}\right|+\xi_{t-1,1}\right)+\mathbf{M}\left(\left|\xi_{t-1}\right|+\xi_{t-1,2}\right)\right)=\frac{3}{2} \mathbf{M}\left|\xi_{t-1}\right|,
$$

и по индукции $\mathbf{M}\left|\xi_{t}\right|=\left(\frac{3}{2}\right)^{t} \mathbf{M}\left|\xi_{0}\right|$.

Аналогично,

$$
\begin{gathered}
\mathbf{M}\left|\xi_{t}\right|^{2}=\frac{1}{2}\left(\mathbf{M}\left(\left|\xi_{t-1}\right|+\xi_{t-1,1}\right)^{2}+\mathbf{M}\left(\left|\xi_{t-1}\right|+\xi_{t-1,2}\right)^{2}\right)= \\
=\mathbf{M}\left|\xi_{t-1}\right|^{2}+\frac{1}{2}\left(\mathbf{M} \xi_{t-1,1}^{2}+\mathbf{M} \xi_{t-1,2}^{2}+2 \mathbf{M}\left|\xi_{t-1}\right|\left(\xi_{t-1,1}+\xi_{t-1,2}\right)\right)= \\
=2 \mathbf{M}\left|\xi_{t-1}\right|^{2}+\frac{1}{2}\left(\mathbf{M} \xi_{t-1,1}^{2}+\mathbf{M} \xi_{t-1,2}^{2}\right)=\frac{5}{2} \mathbf{M}\left|\xi_{t-1}\right|^{2}-\mathbf{M} \xi_{t-1,1} \xi_{t-1,2}
\end{gathered}
$$

Так как

$$
\mathbf{M} \xi_{t-1,1} \xi_{t-1,2}=\frac{1}{2}\left(\mathbf{M}\left(\xi_{t-2,1}+\xi_{t-2,2}\right) \xi_{t-2,2}+\mathbf{M}\left(\xi_{t-2,1}+\xi_{t-2,2}\right) \xi_{t-2,1}\right)=\frac{1}{2} \mathbf{M}\left|\xi_{t-2}\right|^{2},
$$

то

$$
\mathbf{M}\left|\xi_{t}\right|^{2}=\frac{5}{2} \mathbf{M}\left|\xi_{t-1}\right|^{2}-\frac{1}{2} \mathbf{M}\left|\xi_{t-2}\right|^{2}
$$

Общее решение полученного линейного разностного уравнения второго порядка (3) можно представить (см. [2]) в виде

$$
\mathbf{M}\left|\xi_{t}\right|^{2}=C_{1} \lambda_{1}^{t}+C_{2} \lambda_{2}^{t}
$$


где $\lambda_{1}$ и $\lambda_{2}-$ корни соответствующего характеристического уравнения $\lambda^{2}-\frac{5}{2} \lambda+\frac{1}{2}=$ 0 , а константы $C_{1}$ и $C_{2}$ удовлетворяют начальным условиям

$$
\mathbf{M}\left|\xi_{0}\right|^{2}=C_{1}+C_{2}, \quad \mathbf{M}\left|\xi_{1}\right|^{2}=C_{1} \lambda_{1}+C_{2} \lambda_{2} .
$$

Решая эти уравнения, находим:

$$
\begin{gathered}
\lambda_{1}=\frac{5+\sqrt{17}}{4} \approx 2,2807, \quad \lambda_{1}=\frac{5-\sqrt{17}}{4} \approx 0,2192 \\
C_{1}=\frac{\mathbf{M}\left|\xi_{1}\right|^{2}-\lambda_{2} \mathbf{M}\left|\xi_{0}\right|^{2}}{\lambda_{1}-\lambda_{2}}, \quad C_{2}=\frac{\lambda_{1} \mathbf{M}\left|\xi_{0}\right|^{2}-\mathbf{M}\left|\xi_{1}\right|^{2}}{\lambda_{1}-\lambda_{2}} .
\end{gathered}
$$

Отсюда и из (4) следует вторая формула в (2). Утверждение доказано.

Следствие 1. При $t \rightarrow \infty$

$$
\frac{\mathbf{D}\left|\xi_{t}\right|}{\left(\mathbf{M}\left|\xi_{t}\right|\right)^{2}} \rightarrow \infty, \quad \frac{\mathbf{M}\left(\max \left\{\xi_{t, 1}, \xi_{t, 2}\right\}\right)^{2}}{\left(\mathbf{M}\left|\xi_{t}\right|\right)^{2}} \rightarrow \infty
$$

Доказательство. Так как $\left(\frac{3}{2}\right)^{2}=2,25<\lambda_{1} \approx 2,28$, то из (2) следует, что

$$
\left(\mathbf{M}\left|\xi_{t}\right|\right)^{2}=o\left(\mathbf{M}\left|\xi_{t}\right|^{2}\right) \Rightarrow\left(\mathbf{M}\left|\xi_{t}\right|\right)^{2}=o\left(\mathbf{D}\left|\xi_{t}\right|\right), \quad t \rightarrow \infty
$$

что доказывает первое утверждение следствия. Далее,

$$
\mathbf{M}\left(\max \left\{\xi_{t, 1}, \xi_{t, 2}\right\}\right)^{2} \geqslant \frac{1}{4} \mathbf{M}\left|\xi_{t}\right|^{2},
$$

поэтому

$$
\left(\mathbf{M}\left|\xi_{t}\right|\right)^{2}=o\left(\mathbf{M}\left(\max \left\{\xi_{t, 1}, \xi_{t, 2}\right\}\right)^{2}\right), \quad t \rightarrow \infty,
$$

что доказывает второе утверждение.

Следствие показывает, что распределения $\left|\xi_{t}\right|$ и $\max \left\{\xi_{t, 1}, \xi_{t, 2}\right\}$ имеют тяжелые хвосты.

\section{2. Предельная теорема для отношения $\gamma_{t}$}

Введем случайные величины

$$
\gamma_{t}=\frac{\xi_{t, 1}}{\left|\xi_{t}\right|}, \quad t=1,2, \ldots
$$

принимающие значения в отрезке $[0,1]$; очевидно, $\frac{\xi_{t, 2}}{\left|\xi_{t}\right|}=1-\gamma_{t}$, так что по $\gamma_{t}$ и $\left|\xi_{t}\right|$ однозначно восстанавливается $\xi_{t}=\left(\xi_{t, 1}, \xi_{t, 2}\right)=\left(\gamma_{t}\left|\xi_{t}\right|,\left(1-\gamma_{t}\right)\left|\xi_{t}\right|\right)$. Отсюда и из формул (1) следует, что последовательность $\left\{\gamma_{t}\right\}$ образует цепь Маркова с переходными вероятностями

$$
\mathbf{P}\left\{\gamma_{t+1}=\frac{1}{2-x} \mid \gamma_{t}=x\right\}=\mathbf{P}\left\{\gamma_{t+1}=\frac{x}{1+x} \mid \gamma_{t}=x\right\}=\frac{1}{2} .
$$


Равенства (5) определяют переходные вероятности цепи Маркова $\left\{\gamma_{t}\right\}_{t-0}^{\infty}$ с множеством состояний $[0,1]$. Заметим, что цепь $\gamma_{t}$ может попасть в состояние $y \in\left[0, \frac{1}{2}\right)$ только из состояния $x=\frac{y}{1-y}$, а в состояние $y \in\left(\frac{1}{2}, 1\right)$ - только из состояния $x=2-\frac{1}{y}$; в состояние $\frac{1}{2}$ цепь может попасть из состояний 0 и 1 . Таким образом, множество возможных траекторий цепи $\gamma_{t}$ с фиксированным начальным состоянием образует двоичное дерево, и цепь $\gamma_{t}$ никогда не возвращается в состояние, в котором уже побывала.

Теорема 1. Предел

$$
\lim _{t \rightarrow \infty} \mathbf{P}\left\{\gamma_{t} \leqslant x \mid \gamma_{0}=z\right\}=F(x)
$$

существует и не зависит от начального состояния $z \in[0,1]$.

Доказательство. Определим функции $f_{1}, f_{2}:[0,1] \rightarrow[0,1]$ равенствами

$$
f_{1}(x)=\frac{1}{2-x}, \quad f_{2}(x)=\frac{x}{1+x}, \quad x \in[0,1] .
$$

Реализацию цепи Маркова $\left\{\gamma_{t}\right\}_{t=0}^{\infty}$ можно получить как результат последовательного применения к начальному состоянию $\gamma_{0}$ последовательности независимых случайных отображений $f_{\varepsilon_{1}}, f_{\varepsilon_{2}}, \ldots$, где случайные величины $\varepsilon_{1}, \varepsilon_{2}, \ldots$ независимы и $\mathbf{P}\left\{\varepsilon_{t}=1\right\}=\mathbf{P}\left\{\varepsilon_{t}=2\right\}=\frac{1}{2}, t=1,2, \ldots$ :

$$
\gamma_{t}=f_{\varepsilon_{t}}\left(\gamma_{t-1}\right), \quad t=1,2, \ldots
$$

Случайное отображение $f_{\varepsilon_{1}}$ определяет сжимающий оператор на множестве случайных величин, принимающих значения в интервале $(0,1)$ : если $X, Y$ - случайные величины, $\mathbf{P}\{X \in(0,1)\}=\mathbf{P}\{Y \in(0,1)\}=1$, то

$$
\begin{gathered}
\mathbf{M}\left|f_{\varepsilon_{1}}(X)-f_{\varepsilon_{1}}(Y)\right|=\frac{1}{2} \mathbf{M}\left(\left|\frac{1}{2-X}-\frac{1}{2-Y}\right|+\left|\frac{X}{1+X}-\frac{Y}{1+Y}\right|\right)= \\
=\frac{1}{2} \mathbf{M}\left(\frac{|X-Y|}{(2-X)(2-Y)}+\frac{|X-Y|}{(1+X)(1+Y)}\right)= \\
=\frac{1}{2} \mathbf{M}\left(\frac{|X-Y|(5-X(1-Y)-Y(1-X))}{(2+X(1-X))(2+Y(1-Y))}\right) \leqslant \frac{5}{8} \mathbf{M}|X-Y| .
\end{gathered}
$$

Поэтому при любых распределениях начальных значений $\gamma_{0}, \gamma_{0}^{\prime}$ на $(0,1)$ равенства

$$
\gamma_{t}=f_{\varepsilon_{t}}\left(\gamma_{t-1}\right), \quad \gamma_{t}^{\prime}=f_{\varepsilon_{t}}\left(\gamma_{t-1}^{\prime}\right), \quad t=1,2, \ldots,
$$

задают на одном вероятностном пространстве реализации цепей Маркова $\left\{\gamma_{t}\right\}$ и $\left\{\gamma_{t}^{\prime}\right\}$ с переходными вероятностями $(5)$, и при любом $t=1,2, \ldots$ выполняются неравенства

$$
\mathbf{M}\left|\gamma_{t}-\gamma_{t}^{\prime}\right|=\mathbf{M}\left|f_{\varepsilon_{t}}\left(\gamma_{t-1}\right)-f_{\varepsilon_{t}}\left(\gamma_{t-1}^{\prime}\right)\right| \leqslant \frac{5}{8} \mathbf{M}\left|\gamma_{t-1}-\gamma_{t-1}^{\prime}\right|,
$$

откуда следует, что

$$
\mathbf{M}\left|\gamma_{t}-\gamma_{t}^{\prime}\right| \leqslant\left(\frac{5}{8}\right)^{t} \mathbf{M}\left|\gamma_{0}-\gamma_{0}^{\prime}\right| \rightarrow 0, \quad t \rightarrow \infty .
$$

Следовательно, $\mathbf{P}\left\{\gamma_{t} \leqslant x \mid \gamma_{0}=z\right\}-\mathbf{P}\left\{\gamma_{t}^{\prime} \leqslant x \mid \gamma_{0}=z^{\prime}\right\} \rightarrow 0$ при $t \rightarrow \infty$ и любых $x, z, z^{\prime} \in[0,1]$; таким образом, предельное распределение цепи $\gamma_{t}$ при $t \rightarrow \infty$ существует и не зависит от ее начального состояния. 
Поскольку по теореме 1 предельное распределение цепи Маркова $\left\{\gamma_{t}\right\}_{t \geqslant 0}$ существует и не зависит от начального состояния цепи, то для нахождения вида этого распределения можно ограничиться случаем $\gamma_{0}=\frac{1}{2}$. Заметим, что из построения последовательности $\left\{\gamma_{t}\right\}_{t \geqslant 0}$ очевидным образом следует, что если $\gamma_{0}=\frac{1}{2}$, то при любом натуральном $t$ случайные величины $\gamma_{t}$ и $1-\gamma_{t}$ одинаково распределены.

Для описания вида предельной функции распределения $F(x)$ нам потребуются две теоретико-числовые конструкции.

Определение 1. Для двух дробей $\frac{m_{1}}{n_{1}}$ и $\frac{m_{2}}{n_{2}}$ их медиантой называется дробь $\frac{m_{1}+m_{2}}{n_{1}+n_{2}}$.

Заметим, что если $\frac{m_{1}}{n_{1}}<\frac{m_{2}}{n_{2}}$, то $\frac{m_{1}}{n_{1}}<\frac{m_{1}+m_{2}}{n_{1}+n_{2}}<\frac{m_{2}}{n_{2}}$.

Определение 2. Будем называть рядами Минковского $Z_{-1} \subset Z_{0} \subset Z_{1} \subset \ldots$ последовательности, которые строятся рекуррентно:

$$
Z_{-1}=\left\{\frac{0}{1}, \frac{1}{1}\right\}
$$

и если $Z_{k}=\left\{\frac{a_{0}^{(k)}}{b_{0}^{(k)}}, \frac{a_{1}^{(k)}}{b_{1}^{(k)}}, \ldots, \frac{a_{2^{k+1}}^{(k)}}{b_{2^{k+1}}^{(k)}}\right\}, k \geqslant-1$, то

$$
Z_{k+1}=\left\{\frac{a_{0}^{(k)}}{b_{0}^{(k)}}, \frac{a_{0}^{(k)}+a_{1}^{(k)}}{b_{0}^{(k)}+b_{1}^{(k)}}, \frac{a_{1}^{(k)}}{b_{1}^{(k)}}, \frac{a_{1}^{(k)}+a_{2}^{(k)}}{b_{1}^{(k)}+b_{2}^{(k)}}, \ldots, \frac{a_{2^{k+1}-1}^{(k)}+a_{2^{k+1}}^{(k)}}{b_{2^{(k+1}-1}^{(k)}+b_{2^{k+1}}^{(k)}}, \frac{a_{2^{k+1}}^{(k)}}{b_{2^{k+1}}^{(k)}}\right\} .
$$

Нетрудно доказать по индукции, что ряды Минковского являются монотонно возрастающими последовательностями рациональных чисел отрезка $[0,1]$ и для любых двух соседних в ряду Минковского дробей $\frac{m_{1}}{n_{1}}$ и $\frac{m_{2}}{n_{2}}$ справедливо равенство $\left|m_{1} n_{2}-m_{2} n_{1}\right|=1$.

Обозначим через $M_{i}:=Z_{i} \backslash Z_{i-1}, i=0,1,2, \ldots$, множества элементов ряда $Z_{i}$, являющихся медиантами дробей, соседних в ряде Минковского $Z_{i-1}$. Пусть $0<$ $m_{1}^{i}<\ldots<m_{2^{i}}^{i}<1-$ упорядоченные по возрастанию элементы множества $M_{i}$. Из построения последовательности рядов $\left\{Z_{k}\right\}_{k \geqslant-1}$ очевидным образом следует, что $\left|M_{i}\right|=2^{i}$ и $m_{2 j-1}^{i+1}<m_{j}^{i}<m_{2 j}^{i+1}, j=1, \ldots, 2^{i}$.

Известно (см., например, [1]), что $\cup_{k \geqslant 0} Z_{k}=\mathbb{Q} \cap[0,1]$, т. е. объединение элементов рядов Минковского совпадает с множеством всех рациональных чисел отрезка $[0,1]$. Поэтому равенство $\tau\left(\frac{a}{b}\right) \stackrel{\text { def }}{=} \min \left\{k: \frac{a}{b} \in Z_{k}\right\}$ при $\frac{a}{b} \in \mathbb{Q} \cap[0,1]$ корректно определяет функцию $\tau: \mathbb{Q} \cap[0,1] \rightarrow \mathbb{Z}_{+} \cup\{-1\}$.

Утверждение 1. Дроби $\frac{m_{0}}{n_{0}} \quad$ u $\frac{m_{1}}{n_{1}}$ являются соседними в ряду Минковского $Z_{\max \left\{\tau\left(\frac{m_{0}}{n_{0}}\right), \tau\left(\frac{m_{1}}{n_{1}}\right)\right\}}$ тогда и толъко тогда, когда $\left|m_{0} n_{1}-m_{1} n_{0}\right|=1$.

Доказательство. Справедливость утверждения в одну сторону была отмечена выше. Докажем обратное утверждение: если $\frac{m_{0}}{n_{0}}$ и $\frac{m_{1}}{n_{1}}-$ дроби в ряду Минковского $Z_{\max }\left\{\tau\left(\frac{m_{0}}{n_{0}}\right), \tau\left(\frac{m_{1}}{n_{1}}\right)\right\}$ и $\left|m_{0} n_{1}-m_{1} n_{0}\right|=1$, то они соседние в этом ряду.

Не ограничивая общности, будем считать, что $\frac{m_{0}}{n_{0}}<\frac{m_{1}}{n_{1}}$ и $\tau\left(\frac{m_{0}}{n_{0}}\right)<\tau\left(\frac{m_{1}}{n_{1}}\right)$ (доказательство в остальных случаях проводится аналогично). Пусть $k:=\tau\left(\frac{m_{1}}{n_{1}}\right)$. Из 
построения рядов Минковского следует, что $\frac{m_{0}}{n_{0}} \in Z_{k-1}$. Тогда дробь $\frac{m_{1}}{n_{1}}=\frac{a+c}{b+d}-$ медианта двух соседних элементов $\frac{a}{b}, \frac{c}{d}$ ряда $Z_{k-1},(a, b)=(c, d)=1$ и $\frac{a}{b}<\frac{c}{d}$. Далее, поскольку $|a d-b c|=1$, то $m_{1}=a+c, n_{1}=b+d$ и $\frac{m_{0}}{n_{0}}<\frac{m_{1}}{n_{1}}<\frac{c}{d}$.

Покажем, что тогда $\frac{m_{0}}{n_{0}}=\frac{a}{b}$.

Действительно, если $\frac{a}{b}<\frac{m_{0}}{n_{0}}<\frac{c}{d}$, то дроби $\frac{a}{b}$ и $\frac{c}{d}$ не следуют друг за другом в ряду $Z_{k-1}$, что противоречит предположению. Если $\frac{m_{0}}{n_{0}}<\frac{a}{b}<\frac{c}{d}$, то по условию $\frac{m_{1}}{n_{1}}-\frac{m_{0}}{n_{0}}=\frac{1}{n_{1} n_{0}}$, но, с другой стороны,

$$
\frac{m_{1}}{n_{1}}-\frac{m_{0}}{n_{0}}=\frac{a+c}{b+d}-\frac{m_{0}}{n_{0}}=\frac{\left(a n_{0}-b m_{0}\right)+\left(c n_{0}-d m_{0}\right)}{(b+d) n_{0}} \geqslant \frac{2}{n_{1} n_{0}}
$$

так как $a n_{0}-b m_{0}>0$ и $c n_{0}-d m_{0}>0$ - целые числа, и мы опять приходим к противоречию. Следовательно, $\frac{m_{0}}{n_{0}}=\frac{a}{b}<\frac{c}{d}$; тогда дроби $\frac{m_{0}}{n_{0}}$ и $\frac{m_{1}}{n_{1}}$ следуют друг за другом в ряду $Z_{k}$, что и требовалось доказать.

Определение 3. Монотонно возрастающая функиия Минковского ?(x), $x \in[0,1]$, определяется сначала на множестве $\mathbb{Q} \cap[0,1]$ условиями

а) $?(0)=?(0 / 1)=0, \quad ?(1)=?(1 / 1)=1$,

б) если уже определены значения $?(a / b)$ и $?(c / d)$ для двух несократимых и соседних в одном из рядов Минковского дробей $a / b$ и $c / d$, то

$$
?\left(\frac{a+c}{b+d}\right)=\frac{?(a / b)+?(c / d)}{2},
$$

после чего функция ?(·) доопределяется по непрерывности для всех $x \in[0,1]$.

Нетрудно проверить по индукции, что если $Z_{k}=\left\{0=z_{0}^{(k)}<z_{1}^{(k)}<\ldots<z_{2^{k+1}}^{(k)}=\right.$ $1\}$, то

$$
?\left(z_{n}^{(k)}\right)=\frac{n}{2^{k+1}}, \quad n=0,1,2, \ldots, 2^{k+1},
$$

и что на $M_{k}=Z_{k} \backslash Z_{k-1}$ множество значений $?(x)$ совпадает с $\left\{\frac{1}{2^{k+1}}, \frac{3}{2^{k+1}}, \ldots, \frac{2^{k+1}-1}{2^{k+1}}\right\}$.

В [1] доказано, в частности, что функция ? $(x)$ сингулярна на отрезке $[0,1]$, т. е. $\frac{d}{d x} ?(x)=0$ почти всюду на $[0,1]$, и если $x$ - произвольное число из отрезка $[0,1]$, запись которого в форме цепной дроби имеет вид

$$
x=\frac{1}{a_{1}+\frac{1}{a_{2}+\frac{1}{a_{3}+\ldots}}},
$$

где $a_{1}, a_{2}, \ldots$ - целые неотрицательные числа, то

$$
?(x)=\sum_{k \geqslant 1}(-1)^{k-1} \frac{1}{2^{\left(a_{1}+a_{2}+\cdots+a_{k}\right)-1}} .
$$

Теорема 2. Предельная функиия распределения $F(x)$ иепи Маркова $\left\{\gamma_{t}\right\}$ совпадает с функиией Минковского ?(x).

Доказательство. Доказательство теоремы опирается на следующий результат.

Лемма 2. При любом $k=0,1,2, \ldots$ носитель случайной величины $\gamma_{k}$ и множество $M_{k}$ совпадают. 
Доказательство. Доказательство леммы 2 проведем по индукции. Пусть множество $N_{t}$ - носитель распределения случайной величины $\gamma_{t}, t=0,1,2, \ldots$, при $\gamma_{0}=\frac{1}{2}$. Из построения последовательности $\left\{\gamma_{t}\right\}_{t \geqslant 0}$ и определения рядов Минковского следует, что $\left|N_{t}\right| \leqslant 2^{t}$ и $M_{s}=N_{s}, s=0,1,2$.

Пусть утверждение Леммы справедливо при всех $s<k(k>2)$ и $\frac{i}{j}-$ произвольная дробь из $M_{k}$. Покажем, что в этом случае $\frac{i}{j} \in N_{k}$, откуда будет следовать совпадение множеств $M_{k}$ и $N_{k}$, так как $\left|M_{k}\right|=2^{k}$. Заметим, что достаточно рассмотреть случай $\frac{i}{j}<\frac{1}{2}$, поскольку множества $M_{k}$ и $N_{k}$ симметричны относительно середины отрезка $[0,1]$ (если $\frac{i}{j} \in M_{k}$, то $1-\frac{i}{j} \in M_{k}$, и аналогично для $N_{k}$ ).

Так как $\frac{i}{j} \in M_{k}$, то существуют дроби $\frac{m_{1}}{n_{1}} \in M_{l}, \frac{m_{2}}{n_{2}} \in M_{k-1}, l<k-1$, следующие друг за другом в ряду $Z_{k-1}$ (поэтому $\left.\left|m_{1} n_{2}-m_{2} n_{1}\right|=1\right)$ и такие, что дробь $\frac{i}{j}$ является их медиантой, то есть $\frac{i}{j}=\frac{m_{1}+m_{2}}{n_{1}+n_{2}}$. Кроме того, каждый ряд Минковского $Z_{k}, k \geqslant 0$, содержит $\frac{1}{2}$, поэтому если медианта двух его соседних дробей меньше $\frac{1}{2}$, то это же справедливо и для самих дробей (следовательно, выполнены неравенства $\left.2 m_{1}<n_{1}, 2 m_{2}<n_{2}\right)$.

Далее, по предположению индукции $\frac{m_{1}}{n_{1}} \in N_{l}, \frac{m_{2}}{n_{2}} \in N_{k-1}$. Из определения (5) цепи $\left\{\gamma_{t}\right\}_{t \geqslant 0}$ следует, что состоянию $\gamma_{l}=\frac{m_{1}}{n_{1}} \in N_{l}, \frac{m_{1}}{n_{1}}<\frac{1}{2}$, в траектории цепи обязательно предшествует состояние $\gamma_{l-1}=\frac{m_{1} / n_{1}}{1-m_{1} / n_{1}}=\frac{m_{1}}{n_{1}-m_{1}}$, т. е. $\frac{m_{1}}{n_{1}-m_{1}} \in N_{l-1}$, и аналогично $\frac{m_{2}}{n_{2}-m_{2}} \in N_{k-2}$. По предположению индукции $\frac{m_{1}}{n_{1}-m_{1}} \in M_{l-1}, \frac{m_{2}}{n_{2}-m_{2}} \in$ $M_{k-2}$, и

$$
\left|m_{1}\left(n_{2}-m_{2}\right)-m_{2}\left(n_{1}-m_{1}\right)\right|=\left|m_{1} n_{2}-m_{2} n_{1}\right|=1 .
$$

Но тогда по утверждению 1 дроби $\frac{m_{1}}{n_{1}-m_{1}}, \frac{m_{2}}{n_{2}-m_{2}}$ являются последовательными в ряде Минковского $Z_{k-2}$, поэтому их медианта $\frac{m_{1}+m_{2}}{\left(n_{1}-m_{1}\right)+\left(n_{2}-m_{2}\right)}=\frac{i}{j-i}$ принадлежит $M_{k-1}$, а значит, и $N_{k-1}$ (по предположению индукции). Отсюда и из определения (5) цепи $\left\{\gamma_{t}\right\}_{t \geqslant 0}$ следует, что $\frac{i}{j} \in N_{k}$, что и требовалось доказать.

Теперь перейдем к доказательству теоремы 2.

Пусть $F_{k}(x):=\mathbf{P}\left(\gamma_{k} \leqslant x\right)$ - функция распределения случайной величины $\gamma_{k}$. Тогда на $[0,1]$ функция $F_{k}-$ кусочно-постоянна, принимает значения $\left\{\frac{i}{2^{k}} \mid i=\right.$ $\left.0,1,2, \ldots, 2^{k}\right\}$ в точках множества $M_{k} \bigcup\{0 ; 1\}$ и имеет скачки величиной $\frac{1}{2^{k}}$ в элементах $m_{i}^{k}$ множества $M_{k}$. Функция Минковского на элементах множества $M_{k} \bigcup\{0 ; 1\}$ принимает все значения из объединения $\left\{\frac{1}{2^{k+1}}, \frac{3}{2^{k+1}}, \ldots, \frac{2^{k+1}-1}{2^{k+1}}\right\} \bigcup\{0 ; 1\}$ и строго возрастает на интервалах между этими элементами. Возможны несколько случаев:

- на полуинтервале $\left[m_{i}^{k}, m_{i+1}^{k}\right), i \in\left\{1,2, \ldots, 2^{k}-1\right\}$, функция $F_{k}(x) \equiv \frac{i}{2^{k}}$ и $\frac{2 i-1}{2^{k+1}} \leqslant ?(x)<\frac{2 i+1}{2^{k+1}}$, значит, графики функций $F_{k}(x)$ и $?(x)$ пересекаются на этом полуинтервале в точке с ординатой $\frac{i}{2^{k}}$,

- на полуинтервале $\left[0, m_{1}^{k}\right)$ функция $F_{k}(x) \equiv 0$ и $0 \leqslant ?(x)<\frac{1}{2^{k+1}}$, следовательно, $F_{k}(0)=?(0)=0$,

- на полуинтервале $\left[m_{2^{k}}^{k}, 1\right]$ функция $F_{k} \equiv 1$ и $\frac{2^{k+1}-1}{2^{k+1}} \leqslant ?(x) \leqslant 1$, следовательно, $F_{k}(1)=?(1)=1$.

Таким образом, графики $F_{k}(x)$ и $?(x)$ пересекаются в $2^{k}+1$ точках отрезка $[0,1]$ и принимают в точках пересечения значения $\left\{\frac{i}{2^{k}} \mid i=0,1,2, \ldots, 2^{k}\right\}$. С другой стороны, ? $(x)$ принимает эти же значения в точках множества $Z_{k-1}$. Поскольку ? $(x)$ строго монотонна, она принимает любое свое значение ровно один раз, следовательно, 
$\left.F_{k}\right|_{Z_{k-1}}=\left.?\right|_{Z_{k-1}}$. Но $\bigcup_{i \geqslant 0} Z_{i}=\mathbb{Q} \bigcap[0,1]$ - всюду плотное на отрезке $[0,1]$ множество, и $\left.\left(\lim _{k \rightarrow \infty} F_{k}\right)\right|_{\bigcup_{i \geqslant 0} Z_{i}}=\left.F\right|_{\bigcup_{i \geqslant 0} Z_{i}}=\left.?\right|_{\bigcup_{i \geqslant 0}} Z_{i}$. Из совпадения двух монотонных функций (одна из которых непрерывна) в точках всюду плотного множества следует их равенство на всем отрезке:

$$
F(x) \equiv ?(x) \quad \text { при всех } \quad x \in[0,1] .
$$

\section{Список литературы}

1. Salem R., "On some singular monotonic functions which are strictly increasing", Trans. Amer. Math. Soc., 53 (1943), 427-439.

2. Гельфонд А., Исчисление конечных разностей, Москва, ГИФМЛ, 1959.

3. Celler F., Leedham-Green C.R., Murray S., Niemeyer A., O'Brien E.A., "Generating random elements of a finite group", Comm. Alg., 23 (1995), 4931-4948.

4. Diaconis P., Saloff-Coste L., "Walks on generating sets of Abelian groups", Probab. Theory Relat. Fields, 105 (1996), 393-421.

5. Lubotzky A., Pak I., "The product replacement algorithm and Kazdan's property (T)", J. Amer. Math. Soc., 14:2 (2000), 347-363. 